\title{
Energy Efficiency of the IEEE 802.15.4 Standard in Wireless Sensor Networks: Modeling and Improvement Perspectives
}

\author{
Himanshu Sharma \\ Krishna Institute of \\ Engineering \& Technology, \\ Ghaziabad, U.P., INDIA
}

\author{
Vibhav Kumar Sachan \\ Krishna Institute of \\ Engineering \& Technology, \\ Ghaziabad, U.P., INDIA
}

\author{
Syed Akhtar Imam \\ Faculty of Engineering \& \\ Technology, Jamia Millia \\ Islamia, New Delhi, INDIA
}

\begin{abstract}
The IEEE focuses on the physical and MAC layer of Wireless Sensor Networks (WSN) and the Internet Engineering Task Force (IETF) works on Network layer and higher in the seven layer OSI reference model for networking. The IEEE 802.15.4 standard, defines the physical layer (PHY) and medium access control (MAC) sub layer specifications for low-data-rate wireless connectivity with fixed, portable, and moving devices with no battery or very limited battery consumption requirements. In this paper, the energy consumption of a practical Wireless Sensor Network scenario is modeled at the bottom three layers of the traditional networking stack-the physical layer, data link layer and the MAC layer. Using these models, the optimization of energy consumption of WSN nodes is achieved using different modulation schemes to maximize the network lifetime. The approach is to design and simulate a wireless sensor network scenario consisting of 12 nodes in $500 \times 500 \mathrm{~m}$ area and optimize the energy consumption (radio energy + circuit energy) of each node using suitable modulation techniques and reduce the Energy Consumption up to $80 \%$ in a home automation scenario.
\end{abstract}

\section{Keywords}

IEEE 802.15.4 Standard, Wireless Sensor Networks, ,Modulation Techniques, Modeling sensor node energy consumption, Energy Efficiency.

\section{INTRODUCTION}

IEEE 802.15.4 Standard provides for ultra low complexity, ultra low cost, ultra low power consumption, and low data rate wireless connectivity among low-cost devices. The data rate is high enough $(250 \mathrm{~kb} / \mathrm{s})$ to satisfy a set of applications but is also scalable down to the needs of sensor and automation needs $(20 \mathrm{~kb} / \mathrm{s}$ or below) for wireless communications. In addition, one of the alternate PHYs provides precision ranging capability that is accurate to one meter. Multiple PHYs are defined to support a variety of frequency bands including [1]

- $\quad 868-868.6 \mathrm{MHz}$

- $\quad 902-928 \mathrm{MHz}$

- $\quad 2400-2483.5 \mathrm{MHz}$

- $\quad 314-316 \mathrm{MHz}, 430-434 \mathrm{MHz}$, and 779-787 MHz band for LR-WPAN systems in China

- $\quad 950-956 \mathrm{MHz}$ in Japan.

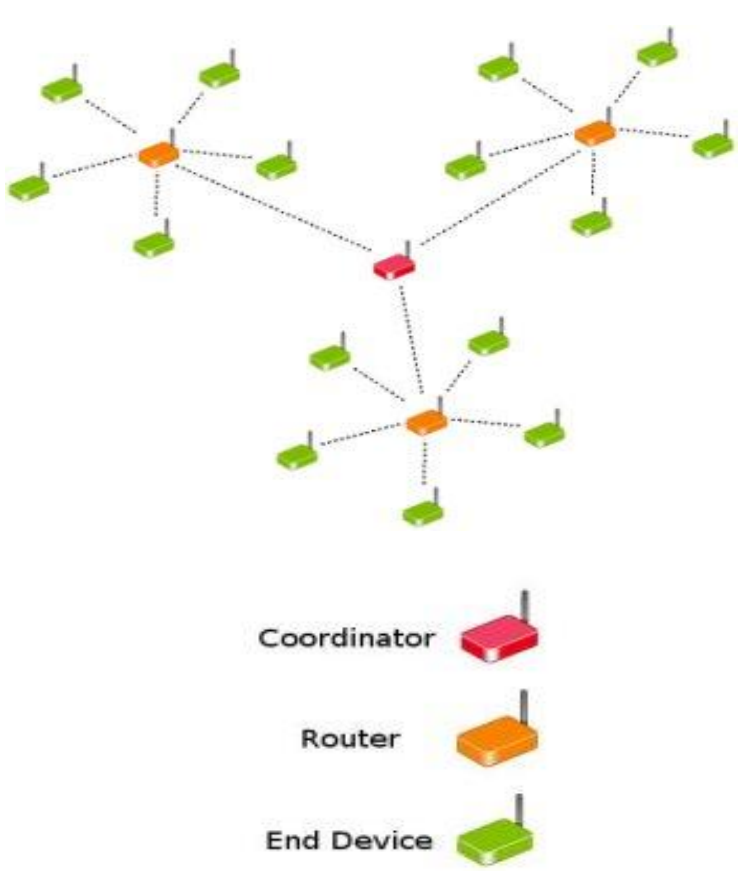

Figure 1: Three kinds of nodes in a Wireless Sensor Network

\subsection{Definitions:}

1.2.1 Coordinator: A device in an low rate wireless personal area network (LR WPAN) that provides synchronization services to other devices in the LR WPAN.

1.2.2 Device: Any entity containing an implementation of the IEEE 802.15.4 medium access control and physical interface to the wireless medium. A device may be a reducedfunction device or a full-function device.

1.2.3 Full-function device: A device capable of operating as a coordinator.

1.2.4 Reduced-function device (RFD): A device that is not capable of operating as a coordinator.

1.2.5 Personal Area Network (PAN) coordinator: A coordinator that is the principal controller of a PAN. An IEEE 802.15.4 network has exactly one PAN coordinator.[1] 


\subsection{Components of the IEEE 802.15.4 WPAN}

A system meeting the requirements to this standard consists of several components. The most basic is the device. Two or more devices communicating on the same physical channel constitute a WPAN. However, this WPAN includes at least one FFD, which operates as the PAN coordinator.[1]

\subsection{Architecture}

The IEEE 802.15.4 design is defined in terms of a number of blocks in order to simplify the standard. These blocks are called layers. Each layer is responsible for one part of the standard and offers services to the higher layers. The interfaces between the layers serve to define the logical links that are described in this standard. An LR-WPAN device comprises at least one PHY, which consists of the radio frequency (RF) transceiver along with its low-level control mechanism, and a MAC sub layer that provides access to the physical channel for all types of transfer. Figure 2 shows these blocks in a graphical representation, which are described in more detail in 1.6 and 1.7.

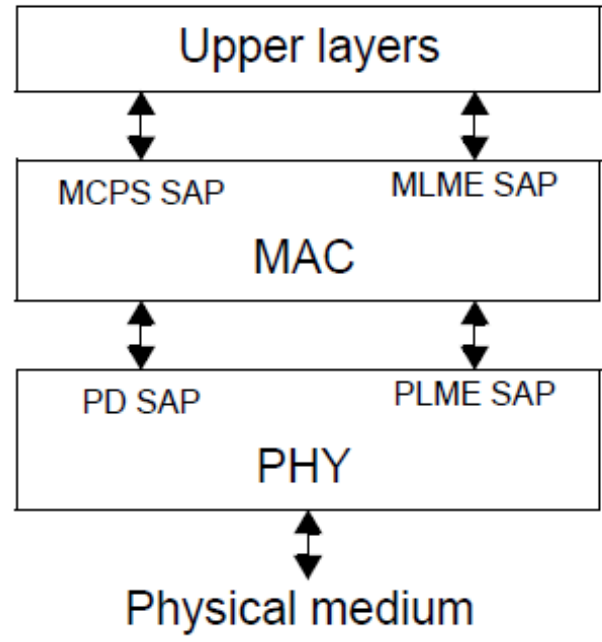

Figure 2: LR-WPAN device architecture

The upper layers, shown in Figure 2, consist of a network layer, which provides network configuration, manipulation, and message routing, and an application layer, which provides the intended function of the device. The definition of these upper layers is outside the scope of this standard [1].

\subsection{Physical layer (PHY)}

The PHY provides two services: the PHY data service and the PHY management service. The PHY data service enables the transmission and reception of PHY protocol data units (PPDUs) across the physical radio channel. The features of the PHY are activation and deactivation of the radio transceiver, ED, LQI, channel selection, clear channel assessment (CCA), and transmitting as well as receiving packets across the physical medium. The UWB PHY also has the feature of precision ranging.[1][2]

\subsection{MAC sub layer}

The MAC sub layer provides two services: the MAC data service and the MAC management service interfacing to the MAC sub layer management entity (MLME) service access point (SAP) (known as MLMESAP). The MAC data service enables the transmission and reception of MAC protocol data units (MPDUs) across the PHY data service. The features of the MAC sub layer are beacon management, channel access, GTS management, frame validation, acknowledged frame delivery, association, and disassociation. In addition, the MAC sub layer provides hooks for implementing applicationappropriate security mechanisms.

\subsection{Power consumption considerations}

In many applications that use these standard, devices will be battery powered, and battery replacement or recharging in relatively short intervals is impractical. Therefore, the power consumption is of significant concern. Battery-powered devices will require duty-cycling to reduce power consumption. These devices will spend most of their operational life in a sleep state; however, each device periodically listens to the RF channel in order to determine whether a message is pending. This mechanism allows the application designer to decide on the balance between battery consumption and message latency. Higher powered devices have the option of listening to the RF channel continuously. In addition to the power saving features of the LR-WPAN system, the UWB PHY also provides a hybrid modulation that enables very simple, non coherent receiver architectures to further minimize power consumption and implementation complexity. [2]

\subsection{Wireless sensor networks}

Wireless sensor networks (WSNs) are typically characterized by battery-powered sensor devices that are expected to operate over prolonged periods of time. Because of the difficulties in replacing the batteries of these devices quickly and regularly and communication being a major source of power drain in such networks, energy-efficient communication protocols are of paramount importance in such networks. To achieve this goal, one needs to address the energy- saving measures in all possible fronts such as physical layer, MAC layer, network layer (e.g., energy-efficient routing) and application layer (e.g., data aggregation)[3]. The aim here in this paper, is to evaluate the performance of modulation schemes in terms of overall energy consumption in the sensor network. In this paper, three different modulation types frequently employed in wireless communications are considered; ASK, BPSK, and OQPSK, and energy minimization scheme for point-to-point wireless communications is derived. The results show that, among these three modulation types, OQPSK has been a preferable choice for WSNs. The minimum energy consumption is numerically analyzed, considering both transmit signal and circuit energy, spent per information bit. After the discussion of ASK, BPSK and OQPSK the work is extended to higher level of modulation scheme viz. MPSK, MQAM, MFSK. Here, the main objective is to derive a suitable Modulation format and the optimum parameters that achieve minimum energy consumption for a given distance between nodes[3].

The remainder of this paper is organized as follows. Section 2 describes related work on WSN; Section 3 describes the modeling of a sensor node energy and then Simulation Parameters in Section 4 and Simulation results in Section 5. Section 6 extends the simulated results to the performance analysis of higher level of Modulations schemes viz. MQAM and MPSK which are widely used in wireless communication. Finally, Section 7 concludes the paper. 


\section{RELATED WORK ON WSN}

Researchers have explored the sensor node energy with different modulation schemes. Chouhan et al. [3] have proposed a framework for energy consumption based design space exploration. Using this framework, the authors have explored various modulations schemes and observed that using modulations saves energy as compared to unmodulated data transmission. E. Shih, S. Cho, F. S. Lee [5] analyze both transmission time and constellation size optimization for MQAM and MFSK (both coded and uncoded), considering both transmission and circuit energy consumption. For both MQAM and MFSK, it has been shown that optimizing transmission time or, equivalently, constellation size, minimizes the total energy used for transmission of information. Sharma and Banerjee [9] has worked on Performance Analysis of narrow band and UWB Modulation Techniques using a Sensor Network Model. This paper extends the work of [12] by considering modulation techniques as performance parameter of WSNs in addition with Error control codes. Here, the three modulation types i.e. ASK, BPSK, and OQPSK, are used to find an energy minimization scheme in IEEE 802.15.4 LRWPAN. Among these three modulation types, OQPSK has been a preferable choice for WSNs because of its less noise interference characteristics. Further, this paper is extended to the study of three most widely used modulation schemes in wireless communication systems, i.e. MQAM, MPSK and MFSK [3] along with suitable Error Correction Codes. The PSK scheme is mostly used in wireless Communication because the probability of error $\left(\mathrm{P}_{\mathrm{e}}\right)$ or Bit Error Rate (BER) in digital sense, is less as compared to ASK scheme in case of PSK the signal to Noise ratio (SNR) is high. A desirable modulation techniques must provide low BER, performs well in multipath and fading conditions, occupies minimum BW, consumes less power, easy to implement and cost effective.

\section{SYSTEM MODEL}

Here, a simplistic physical layer model for the WSN called Zigbee Auto-home scenario with twelve sensor nodes is considered as shown in Fig.4. Here, the network is linear, and the nodes are linearly space and are equidistant to each other. A total of twelve sensor nodes $(1 ; 2 ; 3 ; 4 ; 5 ; 6 ; 7 ; 8 ; 9 ; 10 ; 11$ ;12) are considered which are spreaded in an area of 500x500 meter. All the twelve nodes are homogenous in nature, and they are capable of transmitting as well as receiving signals. Nodes are working in a multihop scenario where node 2 is the Personal Digital Assistant (PDA) node and rest all are sensor nodes of the signal. The signals between links are being affected by the channel, modelled as AWGN, flat Rayleigh fading and lognormal fading in the simulation setup. The signal power has been normalized to unity in all the cases considered. The receivers are assumed to be synchronized. One of the most important aspects in a communication system is the choice of modulation. As it is known that some of the modulation schemes are energy efficient than the others [9], it indeed becomes important in a WSN to use the optimum modulation scheme so as to increase energy efficiency and at the same time maximize data rates as well as minimize the bit error probability.. The main components of a sensor node are shown in fig. 3 which consists following subsystems:
- A Sensor Subsystem

- A Computing/Processing Subsystem

- A Communication Subsystem

- A Power Supply Subsystem

- Location finding Subsystem (optional)

- Mobilizer Subsystem (optional)

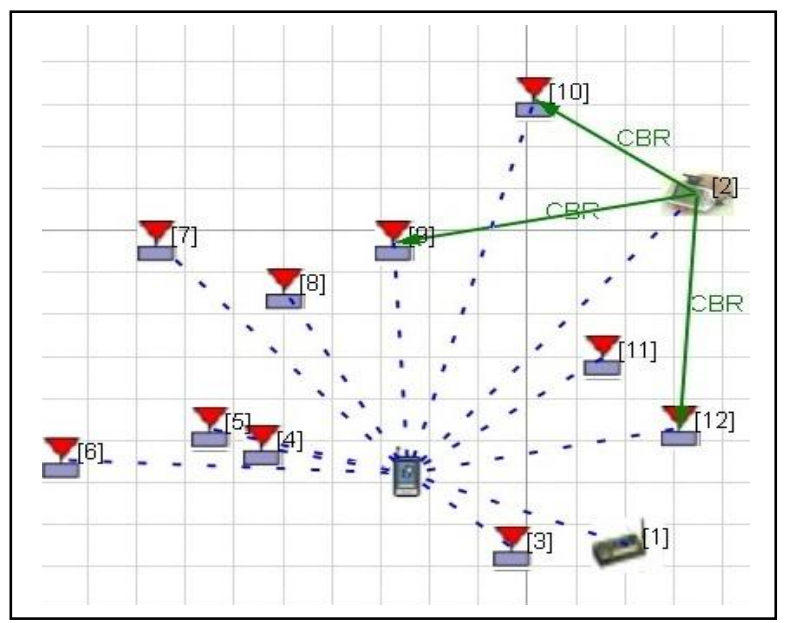

Figure 3 Modelling of a Zigbee Home Automation Scenario with wireless sensor nodes and PDA.

The Energy consumed in case of encoded data transmission is sum of Trans-receiver energy (called radio energy) and energy consumed in Computation circuit energy (called comp. energy). Thus the basic equation can be written as:

$$
E_{n}=\frac{E_{r}+E_{c}}{L}
$$

where, $\mathrm{L}=$ no. of transmitting bits

\section{$\mathrm{E}_{\mathrm{n}}=$ Energy Consumption in Sensor Node}

$\mathrm{E}_{\mathrm{r}}=$ Energy Consumption in Radio Transreceiver Circuit (Transmit+Receive) $\mathrm{E}_{\mathrm{c}}=$ Energy Consumption in Computation Circuit.(CPU+Memory)

thus, the node energy in case of coded data transmission is

$$
E_{n c}=E_{r}+E_{c}
$$

where, $\mathrm{E}_{\mathrm{r}}$ and $\mathrm{E}_{\mathrm{c}}$ are given as

$E_{r}=\frac{P_{O N} T_{O N}+P_{S P} T_{S P}+P_{T R} T_{T R}}{L}$

where, $P_{O N}, P_{S P}$, and $P_{T R}$ are the powers consumed during transceiver ON, SLEEP, and TRANSIENT modes, and $T_{O N}$, $T_{S P}$, and $T_{T R}$ are the Transceiver ON, SLEEP, and TRANSIENT mode durations, respectively. The power consumed in radio during $O N$ mode is the summation of signal transmit power $P_{\mathrm{s}}$ and circuit power consumption $P_{c \text { t. }}$. Thus, the radio energy consumption is

$$
E_{r}=\frac{\left(P_{s}+P_{c t}\right) T_{o n}}{L}
$$

Circuit power consumption $P_{c}$ is contributed by the power amplifier (PA) power $\left(P_{P A}\right)$ and rest of the circuit elements power $\left(P_{c}\right)$ of the transceiver circuit where $\mathrm{P}_{\mathrm{ct}}=\mathrm{P}_{\mathrm{c}}+\mathrm{P}_{\mathrm{PA}}$

$$
\begin{aligned}
& P_{C}=P_{D A C}+2\left(P_{L P F}+P_{F S}+P_{B P F}\right) \\
& +P_{L N A}+P_{I F A}+P_{A D C}
\end{aligned}
$$


where, $P_{P A}, P_{D A C}, P_{L P F}, P_{F S}, P_{B P F}, P_{L N A}, P_{I F A}$, and $P_{A D C}$ are the power consumptions in the respective components. The power consumption in PA is dependent on signal transmit power as

$$
\mathrm{P}_{\mathrm{PA}}=\alpha \mathrm{P}_{\mathrm{S}}
$$

where constant $\alpha$ is related to drain efficiency $\eta$ of $R F$ power amp. with $\boldsymbol{\eta}_{\text {eff }}=1 /(1+\alpha)$. Thus,

$$
E_{r}=\frac{(1+\alpha)\left(P_{s}+P_{c t}\right) T_{o n}}{L}
$$

\subsection{Transmit Signal Energy Model:}

The transmitted power required for sending $\mathrm{L}$ bits can be expressed as given by [8]

$$
P_{s}=\left(\frac{4 \pi}{\lambda}\right)^{2} d^{n} \frac{P_{r}}{G_{t} G_{r}}
$$

This eq. is called friss free space equation.

Here, $\mathrm{d}$ is the distance between the transmitter and receiver;

$\lambda$ is the wavelength of the transmitted signal; $P_{r}$ is the received power; $G_{t}$ and $G_{r}$ are the transmitter and receiver antenna gains, respectively; and $\mathrm{n}$ is the path loss exponent. The received power for an additive white Gaussian noise (AWGN) channel is [8]

$$
P_{r}=S N R b B \frac{N_{O}}{2} N F
$$

where, SNR is the SNR per bit for transmitting the uncoded data, $\mathrm{b}$ is the number of bits per modulation symbol, $\mathrm{B}$ is the bandwidth, $\mathrm{N}_{0} / 2$ is the noise spectral density for an AWGN channel, and NF is the receiver noise figure.

For the coded data, the received power is

$$
P_{r}=S N R_{c} b B \frac{N o}{2} N F
$$

where $\mathrm{SNR}_{\mathrm{c}}$ is the SNR per bit for transmitting $N$ encoded bits for $K$ data bits. In the case of nonbinary ECCs, $N$ and $K$ represent the numbers of symbols. The node energy per information bit for the uncoded system is given by

$$
E_{n}=\frac{(1+\alpha) P_{s} T_{o n}+P_{c t} T_{o n}}{L}
$$

and

$$
E_{n c}=\frac{(1+\alpha) P_{s} T_{o n}+P_{c t} T_{o n}+L \cdot E_{c} \frac{N}{K}}{L}
$$

where $N$ are the encoded bits corresponding to the $K$ data bits. For RS codes, $N$ and $K$ represent number of symbols and it can correct up to $t$ symbols, where $K=N-2 t$. RS code is represented as RS $\left(N, d_{\text {min }}\right)$, where $d_{\text {min }}$ represents the minimum distance of the code and

$$
d_{\min }=2 t+1
$$

To analyze the signal energy variations with the modulation and ECC parameter, any one of the parameters is varied while keeping the others constant. For example, first, the code word length $N$ is kept constant and the signal energy variations are observed with respect to constellation size $b$ and errorcorrecting capability $t$. Next, the error correction capability $t$ is kept constant and signal energy variations with respect to $N$ and $b$ are observed. For this analysis and rest of the paper, the output BER $\left(\mathrm{P}_{\mathrm{b}}\right)$ is assumed to be constant.

\subsection{The Variations in Signal Energy $\left(E_{s}\right)$ with Variations in tand bfor Constant $N$ :}

Using (6) and (8), the transmit signal energy

$$
E_{S}=P_{S} T_{\mathrm{ON}}
$$

can be written as

$\mathrm{E}_{\mathrm{s}}=\mathrm{C}_{1} \mathrm{~b} \mathrm{~T}_{\text {on }} \mathrm{SNR}_{\mathrm{c}}$

where, $\mathrm{C}_{1}$ is constant with respect to the ECC and the modulation scheme and

$$
\mathrm{C}_{1}=\left(\frac{4 \pi}{\lambda}\right)^{2} \mathrm{~d}^{\mathrm{n}} \frac{1}{2 \mathrm{LG}_{\mathrm{r}} \mathrm{G}_{\mathrm{t}}} \mathrm{BNFN}_{0}
$$

Here, the value of Energy $(E)_{s}$ for $M$-ary phase shift keying (MPSK), M-ary quadrature amplitude modulation (MQAM), and M-ary frequency shift keying (MFSK) modulation schemes is calculated. The transceiver "ON" duration and the required SNR per bit depend on the modulation scheme used.

In this paper, the following schemes are considered as :

1. M-ary phase-shift keying (MPSK),

2. M-ary quadrature amplitude modulation (MQAM),

3. M-ary frequency-shift keying (MFSK) modulation schemes.

\subsubsection{For MPSK:[8]}

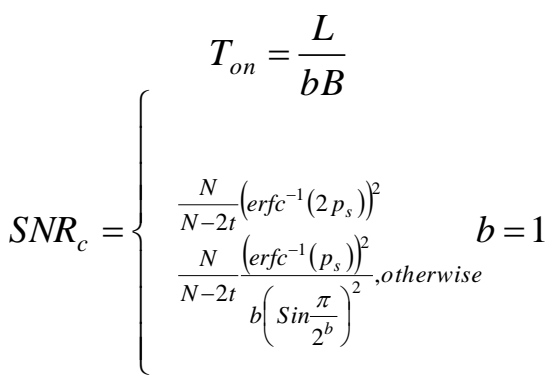

where $p_{s}$ is the channel symbol error probability corresponding to the desired coded symbol error probability $\left(p_{e s}\right)$ or coded bit error probability $\left(p_{e b}\right)$ and computed while computing the coding gain for different RS codes with different modulation schemes [11], [12]. Using (15), (16), and (18), and normalizing the signal energy with respect to the constants, result in

$$
E s=\left\{\begin{array}{l}
\frac{N}{N-2 t}\left(\operatorname{erfc}^{-1}\left(2 p_{s}\right)\right)^{2} \\
\frac{N}{N-2 t} \frac{\left(\operatorname{erfc}^{-1}\left(p_{s}\right)\right)^{2}}{b\left(\operatorname{Sin} \frac{\pi}{2^{b}}\right)^{2}}, \text { otherwise }
\end{array}\right.
$$




\subsubsection{For MQAM:[8]}

$$
\begin{gathered}
T_{o n}=\frac{L}{2 b B} \\
S N R c=\frac{N}{N-2 t} \frac{2\left(2^{b}-1\right)}{3^{b}}\left(e r f c^{-1} \frac{p_{s}}{2\left(1-\frac{1}{\sqrt{2^{b}}}\right)}\right)^{2}
\end{gathered}
$$

Using these relations with (12) and normalizing it with respect to constants, result in

$$
E_{s_{-} n}=\frac{N}{N-2 t} \frac{\left(2^{b}-1\right)}{b}\left(\operatorname{erfc}^{-1} \frac{p_{s}}{2\left(1-\frac{1}{\sqrt{2^{b}}}\right)}\right)^{2}
$$

Table 1.Simulation Parameters:

\begin{tabular}{|l|l|}
\hline Parameters & Values \\
\hline Radio Type & 802.15 .4 Radio \\
\hline Transmission Power (dBm) & 15.0 \\
\hline Packet Reception Model & $\begin{array}{l}\text { PHY 802.15.4 } \\
\text { Reception Model }\end{array}$ \\
\hline Antenna Gain (dB) & 0.0 \\
\hline Antenna Height (meters) & 1.5 \\
\hline Antenna Efficiency (dB) & 0.8 \\
\hline Antenna Mismatch Loss (dB) & 0.3 \\
\hline Antenna Cable Loss (dB) & 0.0 \\
\hline Antenna Connection Loss (dB) & 0.2 \\
\hline Antenna Model & Omni directional \\
\hline Temperature (K) & 290.0 \\
\hline Noise Factor $(\mathrm{dB})$ & 10.0 \\
\hline Energy Model & MICAz Motes \\
\hline
\end{tabular}

\subsubsection{For MFSK:[8]}

$$
\begin{gathered}
T_{o n}=\frac{2^{b} L}{b B} \\
S N R_{c} \leq \frac{N}{N-2 t} \frac{2}{b}\left(\operatorname{erfc}^{-1}\left(\frac{2 p s}{2^{b}-1}\right)\right)^{2}
\end{gathered}
$$

These relations when used with (12) and normalized, result in

$$
E_{s_{-} n} \leq \frac{N}{N-2 t} \frac{2}{b}\left(\operatorname{erfc}^{-1}\left(\frac{2 p s}{2^{b}-1}\right)\right)^{2}
$$

increases, the value of $(N / N-2 t)$ increases and the value of error function term decreases, i.e., although the error correction capability increases, because of the increased redundant bits, the transmit signal energy increases after certain $(t)$. For other modulation schemes also similar trends are observed. As the value of $(\mathrm{N})$ is increased, the signal energy is optimal at a higher value of $(\mathrm{t})$. The value of $(\mathrm{t})$, where signal energy attains the optimal value is independent of the distance. When the constellation size of the modulation scheme is changed, the signal energy is optimal for a different value of (t). For various values of (b) for different modulation schemes and $(\mathrm{N})$, Table I shows the value of $(t)$ for which $E_{s}$ and hence, the transmit signal energy is minimum. Here the energy model of MICAZ motes have been considered for simulations.[18]

\section{SIMULATION OF WSN}

In this approach, a Network Energy Consumption simulator software called Qualnet 5.0 Network simulator [18] which has a research library for wireless sensor networks is used. In this simulator, a sample scenario of home automation (Zigbee Auto Home) is taken and simulated the scenario for $3600 \mathrm{sec}$. in real time within the range of dimension area as $500 \times 500$ meters. The batch experiment for three modulation schemes (ASK, BPSK, and OQPSK) are performed. First the ASK modulation scheme is taken and simulated the scenario and noted down the Energy consumed in all nodes. Then the next modulation scheme is taken in the batch experiment and so on. An important feature of this methodology of research is that this work can be extended for advanced modulation schemes also like MQAM, MPSK etc. for better choice of modulation schemes for WSNs. Table 1 shows the Simulation Parameters i.e. Radio Type, Transmission Power, Packet reception model, Antenna Gain (dB), Noise factor and the type of Energy model used in the sensor nodes.

\section{SIMULATION RESULTS}

In the batch experiment, the simulations results for three modulation scheme are calculated for all nodes. In fig.4, the simulation window of the Qualnet 5.0 network Energy Simulator is shown. A Setup for Performance Analysis of Modulation schemes in WSNs is shown in fig.5. in which each modulation scheme performance is calculated in an energy simulator respectively. In addition to modulation scheme, a suitable ECC can also be used in the setup. Mostly the RS codes prove to be optimized choice for WSNs. The fig. 6 shows Energy Consumed $(\mathrm{mJ})$ by all nodes in the network scenario of Home automation in Transmit, Receive and Idle modes using ASK, BPSK and OQPSK Modulation Schemes respectively. Similarly, Fig.7 shows the total sum of Energy Consumed $(\mathrm{mJ})$ by all nodes in the network scenario in three mode (transmit, receive and idle modes) using ASK, BPSK and OQPSK Modulation Scheme respectively. Here the energy consumed by ASK is $(14.013 \mathrm{~mJ})$ and BPSK has $(25.757 \mathrm{~mJ})$ and OQPSK is $(15.401 \mathrm{~mJ})$.

\section{EXTENSION OF ANALYSIS OF ASK, BPSK AND OQPSK TO MQAM, MPSK AND MFSK SCHEMES}

Based on the performance curves shown in fig.8, 9 and fig.10, and the defining equations for the modulation formats [10], the following statements can be made:

- The bit error rates for BPSK and OQPSK curves have a similar shape in the form of a waterfall which is less than ASK modulation scheme (fig.8). 
- The bit error rates for all the systems decreases monotonically with increasing $B$. The values of $E_{b} / N_{0}$; the defining curves have a similar shape in the form of a waterfall.(fig.10)

- For any value of $\mathrm{E}_{\mathrm{b}} / \mathrm{N}_{0}$, coherent binary PSK, QPSK, and MSK, OQPSK and QAM produce a smaller bit error rate as compared to any other modulation scheme. (fig.10)

- Coherent binary PSK and DPSK need an $\mathrm{E}_{\mathrm{b}} / \mathrm{N}_{0}$ that is 3 $\mathrm{dB}$, less than the corresponding values for conventional coherent binary FSK, to realise the same bit error rate. (fig.10)

- At high values of $\mathrm{E}_{\mathrm{b}} / \mathrm{N}_{0}$, DPSK perform almost as well (to within about $1 \mathrm{~dB}$ ) as coherent binary PSK and conventional coherent binary FSK, respectively, for the same bit rate and signal energy per bit.

- In coherent QPSK, two orthogonal carriers $\sqrt{\frac{2}{T_{b}}} \cos \left(2 \pi f_{c} t\right)$ and $\sqrt{2 / T_{b}} \sin \left(2 \pi f_{c} t\right)$ are used, where the carrier frequency $f_{c}$ is an integer multiple of the symbol rate $1 / \mathrm{T}$, with the result that two independent bit streams can be transmitted simultaneously and subsequently detected in the receiver. (fig.10)

- In fig.9, as the value of $\mathrm{M}$ increases the signal to noise ratio also increases Practically, in wireless communication the value of $\mathrm{M}>8$ is not used.

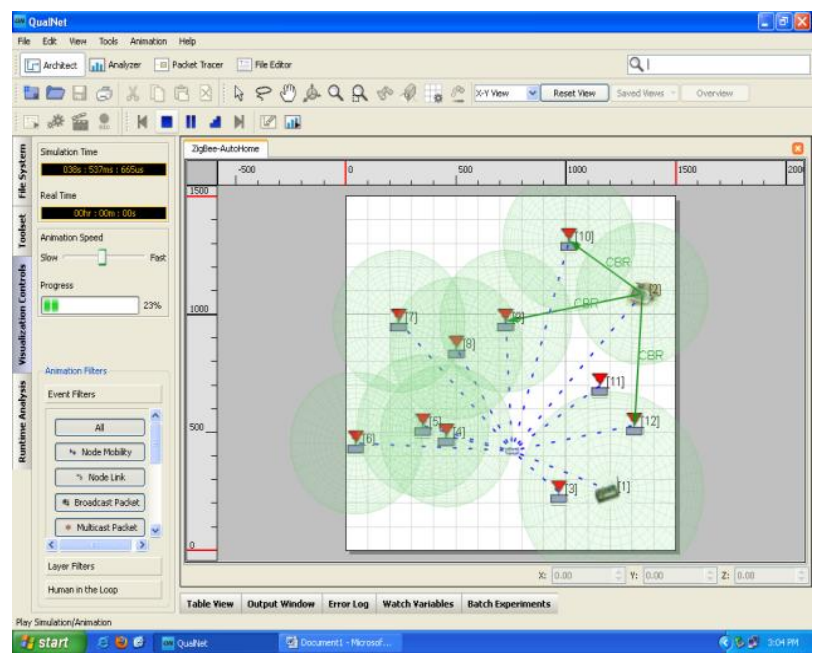

Figure 4 Simulation window of Zigbee Auto-home Wireless Sensor Network with twelve sensor nodes.

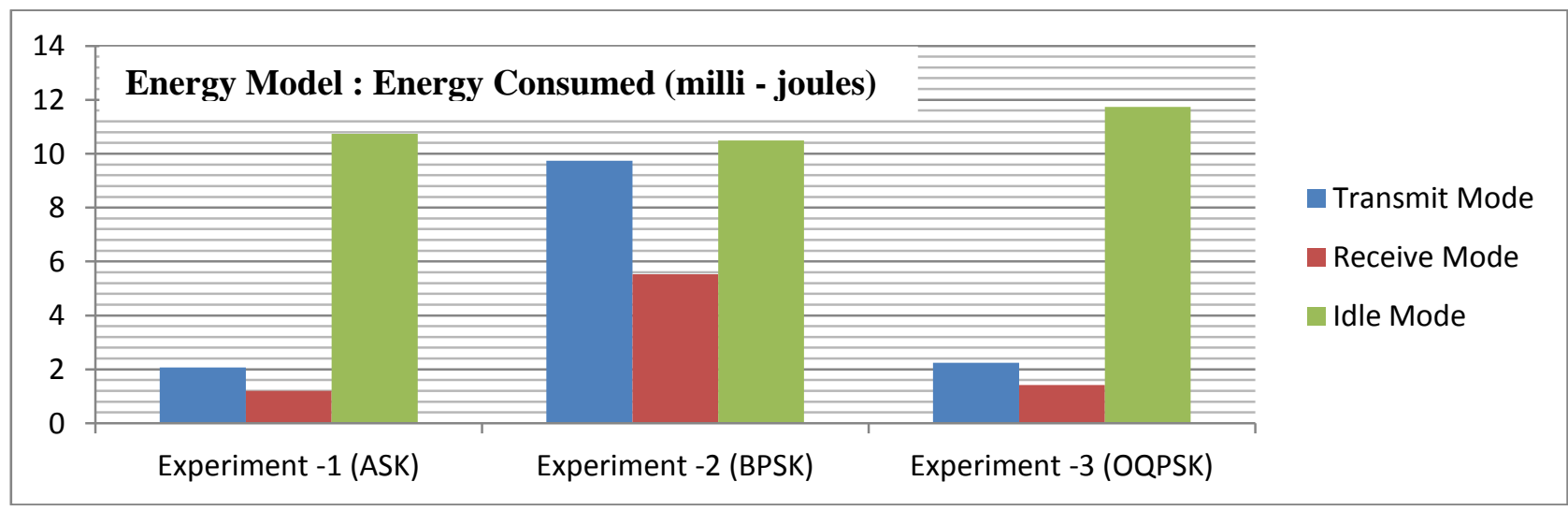

Figure 6 Energy Consumed (mJ) by all nodes in Transmit, Receive and idle modes using ASK, BPSK \& OQPSK Modulation 


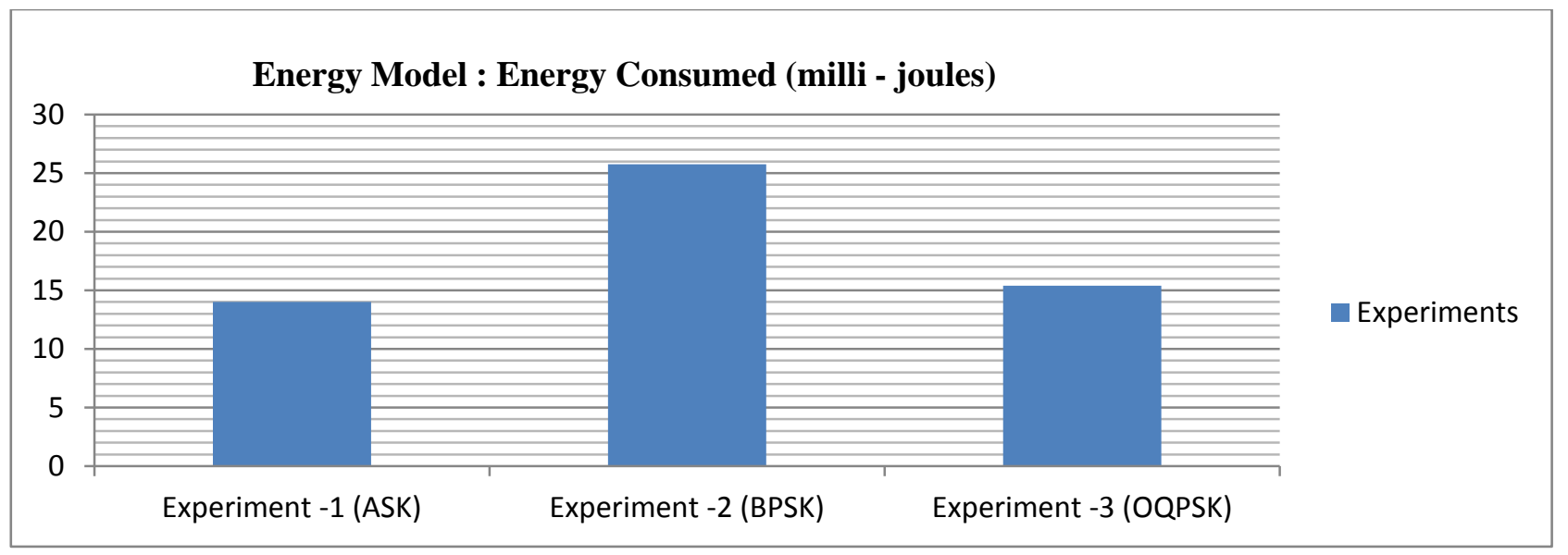

Figure 7 Total Energy Consumed (mJ) by all sensor nodes in three modes of operation (transmit, receive and idle modes) using ASK, BPSK \& OQPSK Modulation schemes

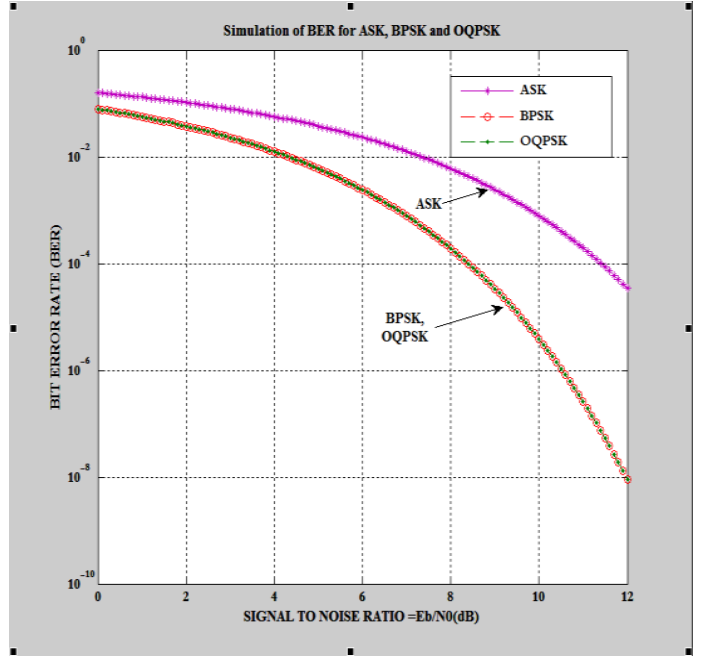

Figure 8 BER performance of ASK, BPSK and OQPSK

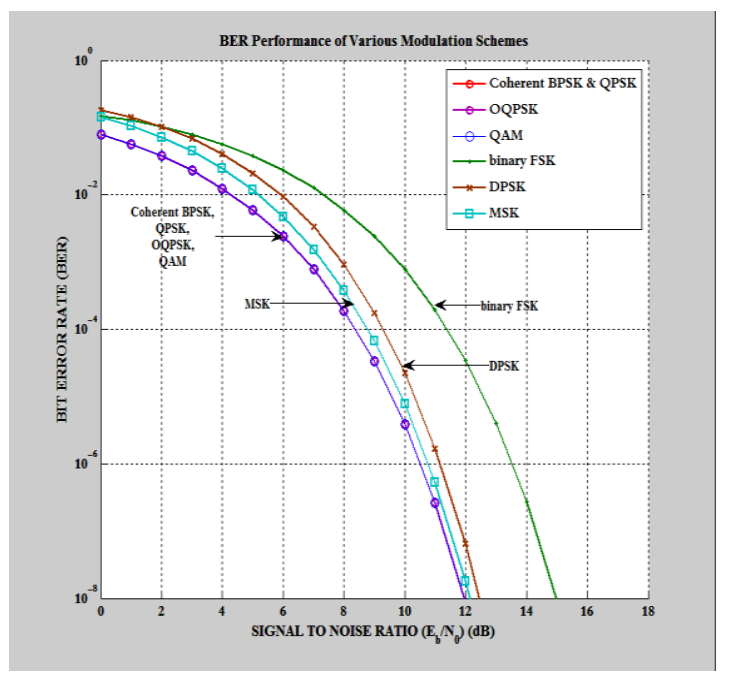

Figure 10 BER performance of various modulation Schemes

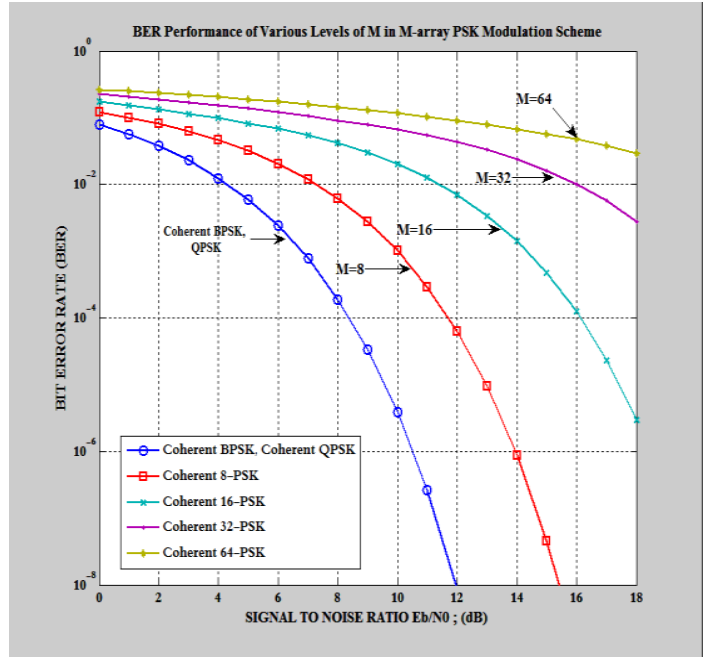

Figure 9 BER performance of various phases in M-phase PSK modulation scheme

\section{CONCLUSION}

From the Simulation results, out of the group of ASK, BPSK and OQPSK, the OQPSK proves to be the best modulation scheme as ASK has high probability of error (BER). Therefore, even if the energy consumption of ASK is (i.e. $14.013 \mathrm{~mJ}$ ) less than OQPSK (i.e. $15.401 \mathrm{~mJ}$ ) but due to its high sensitivity to noise this modulation scheme is rejected and OQPSK is chosen out of the three modulation schemes taken in the batch experiment. The extension of results to higher modulation schemes shows [16] that, for any value of $\mathrm{E}_{\mathrm{b}} / \mathrm{N}_{0}$, coherent binary PSK, QPSK, OQPSK and the QAM produce a smaller bit error rate as compared to any other modulation scheme. Hence, the QAM is cosidered to be the best modulation scheme to be used with WSNs along with RS codes because it has very less noise interference among all modulation schemes.

\section{REFERENCES}

[1]. IEEE Standard Documents for Local and metropolitan area networks-Part 15.4: Low-Rate Wireless Personal Area Networks (LR-WPANs) LAN/MAN Standards Committee IEEE Computer Society Approved 16 June 2011, IEEE-SA Standards Board, Published 5 September 2011. http://www.iee.org. 
[2]. Bruno Bougard, Denis C. Daly "Energy Efficiency of the IEEE 802.15.4 Standard in Dense Wireless Microsensor Networks:Modeling and Improvement Perspectives" Proceedings of the Design, Automation and Test in Europe Conference and Exhibition (DATE'05)15301591/05 IEEE.

[3]. Chouhan, R. Bose, and M. Balakrishnan, "Integrated Energy Analysis of error Correcting Codes and Modulation for Energy Efficient Wireless Sensor Nodes" IEEE transactions on Wireless Communication, vol. 8, no. 10 , October 2009.

[4]. I. F. Akyildiz, W. Su, Y. Sankarasubramaniam, and E. Cayirci, "A survey on sensor networks," IEEE Commun. Mag., pp. 102-114,pp.393-422, August, 2002.

[5]. E. Shih, S. Cho, F. S. Lee, B. H. Calhoun, and A. Chandrakasan, "Design considerations for energyefficient radios in wireless micro sensor networks,"Journal of VLSI Signal Processing Systems for Signal, Image, and Video Technology, vol. 37, no. 1 pp. 7794, 2004

[6]. Sankarasubramaniam, Y., Akyildiz, I.F., and McLaughlin, S.W., "Energy efficiency-based packet size optimization in wireless sensor networks," Proc. 1st IEEE Int. Workshop on Sensor Networks Protocols and Applications SNPA03), (Anchorage, Alaska, USA, 11 May 2003 (held in conjunction with ICC03).

[7]. J. Goldsmith and S. B. Wicker, "Design challenges for energyconstrained ad hoc wireless networks," IEEE Wireless Commun. Mag.,vol. 9, no. 4, pp. 8-27, Aug. 2002.

[8]. Proakis, J. G. , "Digital communications. $4^{\text {th }}$ Edition, New York: McGraw-Hill," 1995.

[9]. Sharma and Banerjee, "Performance Analysis of Energy-Efficient Modulation Techniques for Wireless Sensor Networks “, 978-1-4244-2746-8/08c 2008 IEEE

[10].S. L. Howard, C. Schlegel, and K. Iniewski, "Error control coding in -power wireless sensor networks: when is ECC energy-efficient?" EURASIP J. Wireless Communication Networks., vol. 2006, no. 2, pp. 1-14, 2006.

[11].P. Lettieri, C. Fragouli, and M. B. Srivastava, "Low power error control for wireless links," in Proc. International Conf. Mobile Computing Networking, 1997, pp. 139-150.

[12].Himanshu Sharma, Vibhav Kumar Sachan "Optimization of energy efficiency in Wireless Sensor Networks using Error control codes" Students Conference on Engieering and Systems (SCES-2012), MNNIT, Allahabad, proceeding, pp.79, 2012(Published on IEEE Explore digital library ISBN no. 978-1-4673-0456-6) March 2012.

[13].Polastre, J., Hill, J., and Culler, D, "Versatile low power media access for wireless sensor networks," In Proceedings of ACM international conference on embedded networked sensor systems, Maryland,pp. 95107,2004

[14].H. J. Bergveld, K. M. M. van Kaam, D. M. W. Leenaerts, K. J. P. Philips, A. W. P. Vaassen, and G. Wetkzer, "A low- power highly digitized receiver for 2.4-GHz-band GFSK applications," IEEE Trans.Microw. Theory Tech. vol. 53, no. 2, pp. 453-461, Feb. 2005.

[15] Rappaport, T. S. ,"Wireless communications: principles and practice,"New York: Prentice Hall, 1996.

[16].Symon Haykin, Communication Systems, 4th edition, John Wiley \& Sons, 2001

[17].Anna, H.: Wireless sensor network design (Wiley), 2003.

[18]."Qualnet" 5.0 Scalable Network Technologies, Inc., http://www.scalable-networks.com/qualnet.

[19]."MATLAB” $\quad 7.0 \quad$ (R www.mathworks.com/simulink
2009) 